\title{
Resistenztestung bessert Therapiemanagement
}

\author{
Zunehmend unempfindliche Keime bei der Tuberkulose
}

Neben der Zunahme der HIV-TB-Koinfektionen ist die Verbreitung von medikamentenresistenten Tuberkulosestämmen in Europa besonders besorgniserregend und eine große Herausforderung. Anfang 2008 berichtete die Weltgesundheitsorganisation (WHO) über eine unerwartet deutliche Zunahme medikamentenresistenter Tuberkulosefälle. Fast die Hälfte der Patienten mit multiresistenten Tubekuloseneuerkrankungen weltweit lebt in Indien und China, berichten Hauer et al. Auch Bewohner der Staaten der ehemaligen Sowjetunion haben ein deutlich höheres Risiko für multiresistente Tuberkuloseerkrankungen. Seit 2006 gibt es auch die Form der extensiv resistenten Tuberkulose (,extensively drug-resistant tuberculosis", XDR-TB), die nicht nur gegen die gebräuchlichsten Medikamente Isoniazid und Rifampicin unempfindlich ist, sondern auch Resistenzen gegenüber mindestens einem der Fluorchinolone und einem der drei injizierbaren Zweitrang-Antituberkulotika Amikacin, Kanamycin oder Capreomycin aufweist. Schätzungsweise fünf Prozent der MDR-Fälle haben bereits eine XDR-TB, also etwa 25.000 Fälle, so die Autoren.

Nicht vorhandene bzw. unzureichende Tuberkulosekontrollprogramme, fehlende Ressourcen, inadäquate Präventionsmaßnahmen, aber auch eine verzögerte Tuberkulosediagnostik fördern die weltweiten Ausbreitung der MDR-TB. „Besondere Risikofaktoren für eine MDR-TB sind antituberkulotische Vorbehandlungen, Herkunft aus MDR-TB-Hochprävalenzregionen oder Kontakt zu MDR-TB-Patienten, Gefängnisaufenthalte in manchen Ländern und möglicherweise die HIV-Infektion“, erläutern Hauer et al.

\section{Kombinationstherapie als Standard}

Zur effizienten Behandlung der Tuberkulose muss eine Kombination von Antituberkulotika angewendet werden. Derzeit gilt bei nichtresistenten Lungentuberkulosen die empfohlene Standardchemotherapie aus der initialen zweimonatigen Gabe von vier Erstrangmedikamenten (Isoniazid, Rifampicin, Pyrazinamid und
Ethambutol oder Streptomycin), gefolgt von einer viermonatigen Stabilisierungsphase mit Isoniazid und Rifampicin. „Können eines oder mehrere dieser Erstrangmedikamente wegen Kontraindikationen oder wegen Unverträglichkeiten nicht gegeben werden, verlängern sich die Behandlungszeiten", stellen die Autoren fest. Wichtig ist vor allem bei Verdacht auf eine Medikamentenresistenz eine rasche Sensibilitätstestung, um die Entwicklung weiterer Resistenzen durch ungezielte Therapie zu verhindern. „Hierbei besteht faktisch die Gefahr einer Monotherapie und damit einer weiteren Resistenzentwicklung“, warnen Hauer et al.

\section{Monotherapie führt zu Resistenzen}

Resistenzen können sich dann entwickeln, wenn - bewusst oder unbewusst eine Monotherapie durchgeführt und/ oder keine wirksamen Medikamentenspiegel im Blut erreicht werden. So können Resistenzen und damit Therapieversager oder Rückfälle durch fehlerhafte Medikamentenverordnung, Therapieadhärenzprobleme, Resorptionsstörungen oder mangelnde Medikamentenqualität bedingt sein. Grundsätzlich besteht der Verdacht auf eine Medikamentenresistenz, wenn einer oder mehrere der oben genannten Risikofaktoren vorliegen. Eine endgültige Bestätigung ist nur mit Hilfe - standardisierter und qualitätskontrollierter - bakteriologischer Sensibilitätstestungen möglich. Kulturelle Verfahren der Empfindlichkeitsprüfung, die heute mittels Flüssigkulturen und radiometrischer Verfahren in zwei bis drei Wochen Ergebnisse liefern, sind immer noch der Goldstandard. Schnellere molekularbiologische Methoden zur Detektion verschiedener resistenzvermittelnder Genmutationen stellen laut Hauer et al., besonders für Rifampicin, aber auch Isoniazid, eine enorme Verbesserung dar.

Zur Therapie der komplex resistenten Tuberkulose fehlen bislang randomisierte Studien bzw. evidenzbasierte Daten. Erstund Zweitrangantituberkulotika werden neuerdings in fünf Gruppen eingeteilt, wobei zu den wichtigsten Zweitrangmedikamenten die Fluorchinolone (FQ) zählen.
Antituberkulotisch vorbehandelte Patienten sollen laut WHO-Empfehlung wenigstens drei bislang nicht eingesetzte Medikamente erhalten. Besteht der Verdacht auf Multiresistenz, erhöht sich die empfohlene Anzahl auf mindestens vier potenziell noch wirksame Medikamente, idealerweise auf dem Boden nachgewiesener Empfindlichkeit in der Resistenztestung.

Als wichtigste, die Chemotherapie ergänzende Maßnahmen gelten die Verbesserung der Ernährungs- und sozialen Situation. In bestimmten Situationen kann ein zusätzlicher operativer Eingriff bei MDR- oder XDR-Tuberkulose indiziert sein. Hauer et al. zählen dazu nicht ausreichend zur Verfügung stehende wirksame Medikamente, Ausbleiben der kulturellen Konversion, persistierende Kavernen, weitgehend lokalisierter Befall und fehlende funktionelle Kontraindikationen. Kontrollierte Studien dazu fehlen jedoch.

\section{Behandlung der M/XDR-TB auch in Deutschland unbefriedigend}

Die Behandlungsergebnisse von M/XDRTB seien, so die Autoren, selbst in Deutschland oftmals unbefriedigend. Forderungen an das Gesundheitssystem umfassen daher neben besseren Verfahren zur Tuberkulosediagnostik (raschere Resistenztestung), einer Behandlung in erfahrenen Zentren, einer konsequenten Therapie-Überwachung sowie adäquater Infektionskontrolle auch dringend die Entwicklung neuer, hochwirksamer Medikamente gegen die Tuberkulose. Hinsichtlich der Isolierung bei Ansteckungsfähigkeit zur optimalen Betreuung komplex resistenter TB-Patienten bedarf es der Klärung der Kostenübernahme, stellen Hauer et al. fest: „Denn diese Maßnahme ist eine entscheidende Voraussetzung dafür, dass Übertragungen auf andere Menschen und damit Neuerkrankungen an resistenter TB mit ihren hohen direkten und indirekten Krankheitskosten verhindert und damit langfristig Kosten eingespart werden."

Hauer B, Castell S, Loddenkemper R (2011) Resistente Tuberkulose. Zunehmende Probleme und Lösungsansätze, Pneumologe; 8:25-31 